\title{
Photoacoustic response induced by nanoparticle-mediated photothermal bubbles beyond the thermal expansion for potential theranostics
}

Siqi Wang

Lei Fu

Jing Xin

Sijia Wang

Cuiping Yao

Zhenxi Zhang

Jing Wang 


\title{
Photoacoustic response induced by nanoparticle-mediated photothermal bubbles beyond the thermal expansion for potential theranostics
}

\author{
Siqi Wang, ${ }^{\dagger}$ Lei Fu, ${ }^{\dagger}$ Jing Xin, Sijia Wang, Cuiping Yao, Zhenxi Zhang, ${ }^{*}$ and Jing Wang* \\ Xi'an Jiaotong University, School of Life Science and Technology, Key Laboratory of Biomedical Information Engineering of Ministry of Education, \\ X'an, China
}

\begin{abstract}
Photoacoustic responses induced by laser-excited photothermal bubbles (PTBs) in colloidal gold solutions are relevant to the theranostics quality in biomedical applications. Confined to the complexity of nonstationary, multiscale events, and multiphysical parameters of PTBs, systematic studies of the photoacoustic effects remain obscure. Photoacoustic effects mediated by PTB dynamics and a physical mechanism are studied based on a proof-of-principle multimodal platform integrating side-scattering imaging, time-resolved optical response, and acoustic detection. Results show excitation energy, nanoparticle (NP) size, and NP concentration have strong influence on photoacoustic responses. Under the characteristic enhancement regime, the photoacoustic signal amplitude increases linearly with excitation energy and increases quadratically with the NP diameter. As for the effects of the NP concentration (characterized by absorption coefficient), a higher photoacoustic signal amplitude is generally induced by a dense NP distribution. However, with an increase in the NP size, the shielding effect of NP swarm prevents the increase of photoacoustic responses. This study presents experimental evidence of some key physical phenomena governing the PTB-induced photoacoustic signal generation in gold NP suspensions, which may help enrich theranostic approaches in clinical applications by rationalizing operation parameters. ( ) The Authors. Published by SPIE under a Creative Commons Attribution 3.0 Unported License. Distribution or reproduction of this work in whole or in part requires full attribution of the original publication, including its DOI. [DOI: 10.1117/1.JBO.23.12.125002]

Keywords: photoacoustic signal; plasmonic nanoparticle; vaporization; theranostics.
\end{abstract}

Paper 180474RRR received Aug. 1, 2018; accepted for publication Nov. 26, 2018; published online Dec. 14, 2018.

\section{Introduction}

Plasmonic nanoparticles (NPs) have received extensive attention as exogenous cellular agents because of their good biocompatibility, relative safety in vivo, ease of bioconjugation with biomarkers, and superior optical properties. ${ }^{1-4}$ These factors benefit the potential applications of NP-based photoacoustic detection, including biomedical imaging, ${ }^{5,6}$ in vivo image-guided theranostics, ${ }^{7,8}$ and in vitro biosensing. ${ }^{9,10}$ In conventional photoacoustic imaging, noninvasive and biologically safe modality is indispensable, where photoacoustic signals induced by thermoelastic mechanism might satisfy this demand exactly. ${ }^{11}$ In this modality, photons absorbed by NPs are converted to heat with temperature rise, leading to thermoelastic expansion and emission of acoustic transients, which can be probed by an ultrasound transducer and then used for constructing photoacoustic images. ${ }^{12,13}$ The imaging quality is strongly influenced by light-sound conversion efficiency, which is correlated directly with the optical and thermoelastic properties of the absorbing medium. ${ }^{14,15}$ The introduction of exogenous efficient plasmonic NPs can improve imaging contrast, which is seriously impeded by the background signals from blood due to comparable absorption properties with contrast agents. Multilaser irradiation modality ${ }^{13,16}$ and the cluster effect of $\mathrm{NPs}^{7,17}$ can be utilized to enhance acoustic signals through localized thermal coupling. The shell-core structure of $\mathrm{SiO}_{2} / \mathrm{ZnTPP}$ encapsulated metal $\mathrm{NP}^{13,18}$ has been designed to

\footnotetext{
*Address all correspondence to Zhenxi Zhang, E-mail: zxzhang@xjtu.edu.cn; Jing Wang, E-mail: wangjing@xjtu.edu.cn

tThe authors contributed equally to this work.
}

realize the nonlinear enhancement of photoacoustic signals because the composite structure can reduce interfacial thermal conductance, thereby leading to confined heat accumulation.

Although outstanding designs of excitation modalities and NP structures have been developed, photoacoustic signals remain limited to the dominant mechanism of thermal expansion, which has been considered the least efficient mechanism of optical-to-acoustic energy conversion ${ }^{11,19}$ with relatively low photoacoustic signal. With the occurrence of integrated diagnosis and treatment (referred as theranostics), ${ }^{20-22}$ a mechanism of medium vaporization to motivate an efficient photoacoustic signal has been put forward. Moreover, when the excitation energy increases to a specific level, the interfacial temperature will arrive rapidly at a threshold state where the adjacent medium starts to evaporate and provoke the generation of photothermal bubbles (PTBs). One example of the adjacent medium is water, which is the most important liquid of human organism (e.g., the main component of interstitial fluid, plasma of blood, and intercellular fluid) and thus is frequently used as the background solvent. The threshold temperature required for the water vaporization around the plasmonic ( $\mathrm{Au}) \mathrm{NP}$ is distinctly different from that of the explosive boiling level for pure water (373 K at ambient pressure). The medium vaporization was predicted to be triggered at the $80 \%$ to $85 \%$ of the critical temperature, i.e., 517 to $550 \mathrm{~K}$, based on previous experimental studies using the pump-probe laser system ${ }^{23}$ and the $\mathrm{x}$-ray scattering technique. ${ }^{24}$ Once the phase transition of the water occurs, medium vaporization becomes the primary mechanism to produce photoacoustic signals whether in the case of short-pulse $(30 \mathrm{ps})^{20}$ or longpulse excitation in this work (5 ns), where the signal correlates 


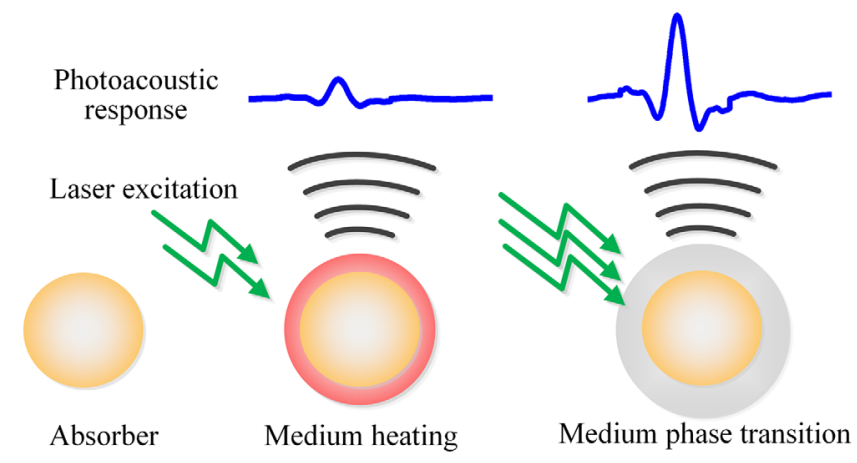

Fig. 1 Schematic of the photoacoustic signals induced by thermal expansion and medium vaporization.

with the NP-based bubble dynamics. Once the bubble around a heated absorber is generated, the photoacoustic effect is always "giant," no matter with what kind of the absorber (endogenous chromophores or exogenous NPs), ${ }^{25-27}$ as shown schematically in Fig. 1. Compared with other vapor bubbles (e.g., induced by endogenous absorbers through bulk heating ${ }^{28}$ ), the PTB induced by plasmonic NPs is uniquely provided with nanoscale effect due to a high level of localized thermal field. This effect could thermally insulate the outer environment from the high-temperature NP, thus reduce the risk and range of thermal damage to the minimum. ${ }^{28}$ Therefore, we confined our work to the plasmonic NP (particularly, the Au NP) in background solvent of water.

As regards the PTB-based theranostics, the effectiveness of the PTB-induced therapy is the prerequisite of establishing the corresponding photoacoustic diagnosis system. In turn, the photoacoustic response could be used for monitoring therapy processes, evaluating therapeutic effects, and regulating laser doses for real-time integrated diagnoses and treatments. Meanwhile, the medium vaporization to provoke PTB generation has also been demonstrated as the key mechanism behind therapy processes in many clinical applications. For example, Xiong et al. $^{29}$ experimentally showed that delivering siRNA across the plasma membrane of targeted cells when nanoscale pores are created by localized mechanical stress of PTB is more efficient than direct heating. More convincing evidences have tended to support that cell death during pulsed laser treatments is dominated by the mechanical effect of PTB dynamics ${ }^{30-32}$ instead of photothermolysis. For microscale vascular applications, Wan's group ${ }^{33}$ recently showed the occlusion and rupture of capillary bifurcations can be regulated successfully by the initialization of droplet vaporization in an ex vivo rat mesentery model. And understanding the transient bubble dynamics after vaporization is of paramount importance for the application of this vaporization-based approach in gas embolotherapy therapies. As a summary, the bubble-based therapy spans comprehensively nanoscale reversible damages in gene therapies ${ }^{29,34}$ to microscale vascular applications. ${ }^{11,33,35}$ To integrate the dual effects of bubble-based diagnostics and therapeutics, a thorough study of the relationship between bubble dynamics, represented by the (maximum) bubble dimension with multistage size, and photoacoustic responses, which are connected with the therapeutic and diagnostic effects, is necessary.

Although massive outstanding achievements related to PTBgenerated photoacoustic signals have emerged, ${ }^{20,26,36}$ some key problems still need to be resolved: first, the range of laser energy in use is very limited, where only the nanoscale bubble occurs.
This situation could not meet rising demands for bubble applications with multiscale size. Knowledge of the physical mechanism behind the NP-mediated PTB can help crack the internal information of bubble kinetics through exterior photoacoustic responses, especially for in vivo studies. Second, influencing factors, which are associated closely with theranostics, are still insufficient, for example, NP size and concentration. Whether an interactive effect exists between these key factors remains to be answered. Third, in the real applications of NP-based therapies, the NP stays in an ensemble state modeling with NP suspension (another typical NP state, the NP clusters, also exists but is not included in this paper). The collective behavior of NP-based bubble population as well as the dynamic change of thermophysical properties during the bubble formation will complicate and even hinder the theoretical prediction of photoacoustic effect. Thus, building the relationship between photoacoustic signals and NP suspensions systematically under tunable excitation conditions remains a challenge. To improve the difficult dilemma above, this work conducted a comprehensive research on the behavior of plasmonic metal NP (e.g., gold nanospheres) excited by pulsed laser through a self-built and proof-of-concept multimodal platform. The system contains a pump-and-probe module, which could realize the single-channel excitation, as well as the multichannel detection. The core component of the pump module is composed of the most available type of 5-ns pulsed laser with a wavelength of $532 \mathrm{~nm}$. On the basis of the platform, the effects of operation conditions (including excitation energy, NP size, and concentration) were studied thoroughly, which complied substantially and ultimately with the realistic demand of medical applications. Hereinto, to probe into the mechanism of multiscale responses of photoacoustic signals to excitation energy, a pump laser was modulated to yield an upper limit high enough to induce an optical breakdown.

\section{Materials and Experimental Setup}

\subsection{Experimental System and Principle}

The configuration of the experimental setup is shown in Fig. 2. A pump laser with a wavelength of $532 \mathrm{~nm}$ and a pulse duration of $5 \mathrm{~ns}$ was provided by a Q-switched, frequency-doubled $\mathrm{Nd}$ : YAG (Q-smart 450, Quantel) laser system. The laser energy was adjusted by a motorized half-wave $(\lambda / 2)$ plate and recorded by a calibrated pyroelectric energy meter (PE-DIF-C, Ophir Optronics). A cooled EMCCD (Ixon ultra897, Andor) was set perpendicular to the direction of pump beam propagation. A digital delay generator (DG645, Stanford Research Systems Inc.) was adopted to synchronize the pump laser with the EMCCD. The EMCCD provided the side-scattering imaging of the side-deflection signal (SDS) collected by a $10 \times$ objective. A set of neutral density filters was placed in front of the imaging device to protect the light-sensitive EMCCD from the highintensity pump laser. The diameter of the focal spot $(16.8 \mu \mathrm{m})$ was determined by fitting the Gaussian function to the spatial intensity distribution measured through the EMCCD. To eliminate the influence of the scattering signal from the NPs, the NP suspension was also illuminated with 532-nm continuous wave (CW) laser when the pulsed laser was off. The average energy of the CW laser was adjusted to the order of magnitude of pulsed energy but far below the threshold of the bubble generation. The side-scattering imaging method facilitated the precise 


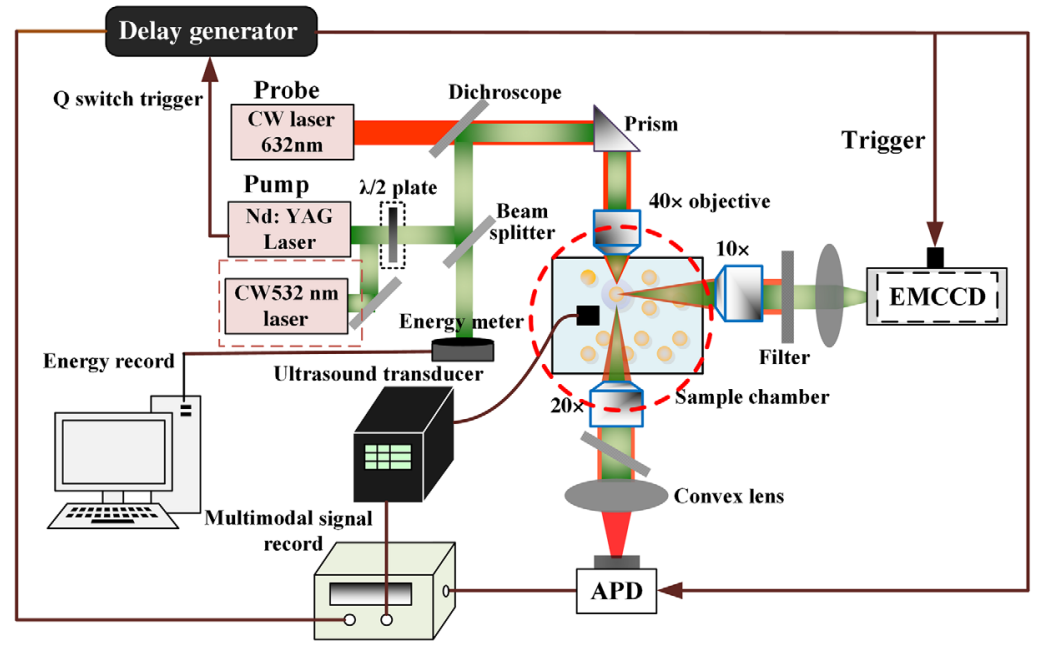

(a)

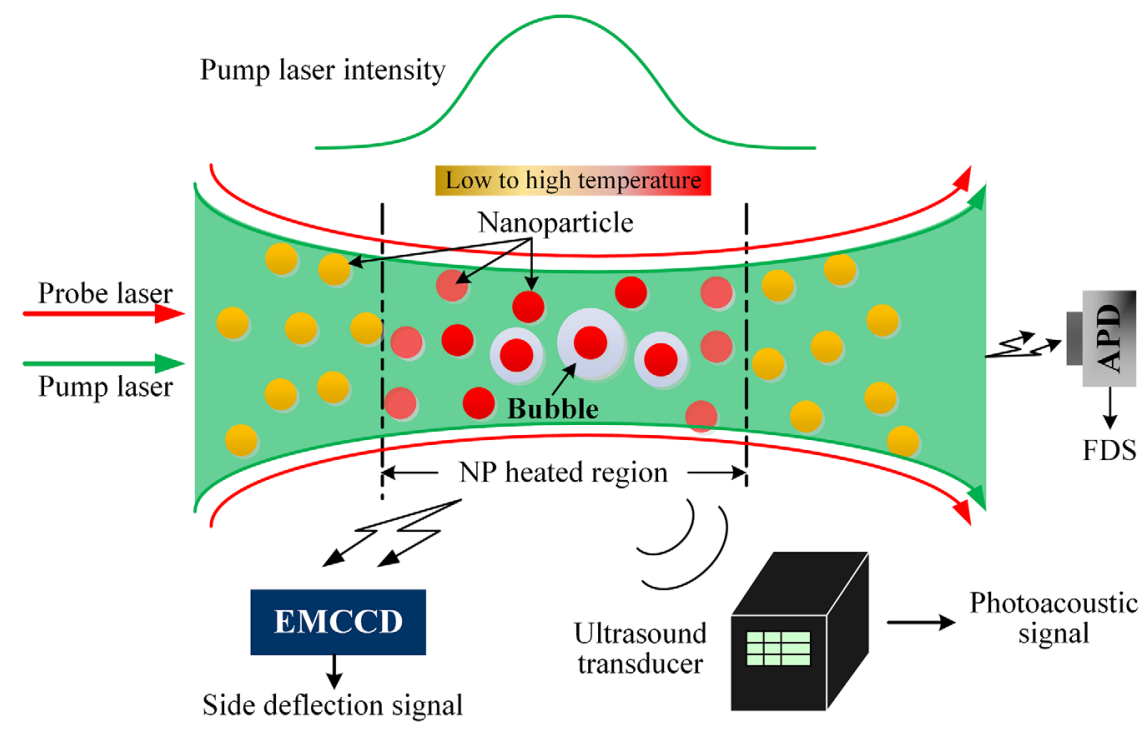

(b)

Fig. 2 Schematic of the experimental system and principle: (a) The multimodal experiment setup and (b) identification of the multimodal signals for NP-based plasmonic bubble generation.

identification of the laser-induced bubbles and provided their spatial distribution.

A CW HeNe laser (HNL 020RB, Thorlabs) with a Gaussian $\mathrm{TEM}_{00}$ profile was used as the probe source to detect the forward beam-deflection signal. The beam was adjusted to be confocally aligned with the pump laser. The two beams went directly through a long-distance $(\sim 0.13 \mathrm{in}$. and $40 \times)$ objective lens and then irradiated into the customized sample chamber with high transmittance. The transmitted probe laser through the suspensions in the chamber was collected by a $20 \times$ objective lens and projected onto an AC-coupled avalanche photodiode (APD) (FEMTO, $25 \mathrm{kHz}$ to $200 \mathrm{MHz}$ bandwidth), in front of which a set of dichroscopes was used to extract the pump irradiation from the transmitted light. An ultrasound transducer with a central bandwidth of $25 \mathrm{MHz}$ (V324-N-SU, Olympus) was immersed into the chamber to detect the photoacoustic signal. The generated acoustic response was the third approach to identify the bubble occurrence. A transducer with an outer diameter of $0.37 \mathrm{in}$. was positioned perpendicularly to the horizontal plane of the chamber, and the active surface was submerged into the suspension with a distance of approximately $0.17 \mathrm{in}$. from the laser focus. An ultrasound pulser/receiver (5662PR, Olympus) was connected to the transducer through a waterproof BNC socket (BCU-58-6W, Olympus) for signal amplification and filtering. The high-speed APD and pulser/receiver were connected to a four-channel digital oscilloscope with a sampling frequency of $5 \mathrm{GHz}$ (PTE1204, Rohde \& Schwarz) for signal recording.

Details about the detection principle of NP-mediated PTBs are shown below. For the plasmonic metal NPs suspended in a weakly absorbing solvent excited by a pulsed laser, a multistage energy-conversion pathway existed. At low laser energy, the excitation of NPs will lead to instantaneous temperature accumulation in the electron-lattice system, along which the heat dissipation to the surroundings will result in a rapid temperature rise and a subsequent outgoing thermoacoustic wave. When the energy reached a threshold to provoke the medium vaporization, photoactivated PTBs will occur around the overheated 
NPs. The PTBs will then undergo a rapid expansion and collapse, resulting in giant photoacoustic signals, which are synthetic (dual) responses triggered by the thermal expansion through localized heating and the thermomechanical transients via vaporization. The dynamics of bubble and photoacoustic wave can also give rise to a mechanical disturbance on the refractive index of the adjacent liquid. Additionally, a thermally modulated gradient of the refraction index may occur because of heat transfer to the surrounding liquids. As shown in Fig. 2(b), due to the existing media heterogeneities activated by the above, the light deflection signal can be detected. Hereinto, the forwarding deflection signal (FDS) is usually considered with the optimum deflection efficacy. ${ }^{36}$ For a single free bubble, the evolution of expansion and collapse phase is symmetrical, which results in a high degree of symmetry of FDS. By contrast, in laser-heated NP without bubble generation, an asymmetric FDS could be observed, with a relatively slow coming-back process due to thermal diffusion ${ }^{37}$ and the thermal lensing effect. $^{38}$ The significant differences between the two FDS profiles could distinguish the PTB signal from the purely photothermal ones. PTB-induced FDS was a bubble-specific and timeresolved signal, characterized by bubble oscillation magnitude and oscillation time (indicated by $T_{\mathrm{osc}}$ ). The former is associated in some cases but not always with bubble size and is very sensitive to the bubble location. The latter, $T_{\text {osc }}$, is considered approximately linearly proportional to the maximum bubble size $R_{\max }$ according to Rayleigh's equation, ${ }^{39,40}$ which is a basic equation to describe the bubble dynamics as follows:

$R_{\max }=\frac{T_{\mathrm{osc}}}{1.83}\left(\frac{P_{s}-P_{v}}{\rho}\right)^{0.5}$

where $\rho=998 \mathrm{~kg} / \mathrm{m}^{3}$ is the density of water, $P_{\mathrm{s}}$ is the hydrostatic pressure, and $P_{\mathrm{v}}$ is the vapor pressure inside the bubble $\left(P_{\mathrm{s}}=0.1 \mathrm{MPa}\right.$ and $P_{\mathrm{v}}=2330 \mathrm{~Pa}$ at $\left.293 \mathrm{~K}\right)$. No matter in what case (single bubble or multibubbles), the FDS could only estimate the lifetime $T_{\text {osc }}$ of the largest one in the confined laser-irradiated region. ${ }^{36}$ However, time-resolved FDS sometimes failed to represent the bubble oscillation in the case with excitation energy close to the PTB occurrence threshold (indicated by $E_{\mathrm{th}}$ ), especially for small NPs. ${ }^{41} E_{\mathrm{th}}$ is defined as excitation energy resulting in a $50 \%$ probability of PTB generation. ${ }^{32}$ Hence, a multimodal detection technique was further adopted for a precision detection, which will be detailed in Sec. 3.1.

Very high excitation energy might result in the optical breakdown of the medium, where a competition might exist between the occurrences of PTB and the cavitation bubble induced by optical breakdown. Thus, the measured excitation energy $(250 \mu \mathrm{J})$ in our work corresponding to the threshold of optical breakdown, $E_{\mathrm{CBO}}$ (CBO indicated cavitation bubble occurrence), was set as the upper limit of the focused energy in the experiments. $E_{\mathrm{CBO}}$ was a statistical concept, defined as the incident pulse energy to provoke a $50 \%$ probability of bubble generation in the NP-free solutions. Above the excitation energy $E_{\mathrm{CBO}}$, the thermomediated PTB occurs with low frequency. The optical breakdown-mediated cavitation bubble was quite distinct from that of the PTB. ${ }^{42}$ Here, we only focused on the breakdown threshold energy, instead of the cavitation effect itself.

\subsection{Material Preparation}

Gold NPs with diameters of 10, 15, 20, 40, 50, and $60 \mathrm{~nm}$ (BBI Solution Inc.) were adopted. The excitation spectrum, describing the optical density (OD), was obtained through the spectrophotometer (V-550 UV/VIS, Jasco, Japan), which was in fact the sum of absorption and scattering. According to the BeerLambert's law, the measured OD linearly depended on the NP concentration: ${ }^{43}$

$\mathrm{OD}=\frac{\pi \cdot D^{2} \cdot Q_{\mathrm{ext}} \cdot d_{0} \cdot c}{2.303 \times 4}$,

where excitation efficiency $Q_{\text {ext }}$ could be calculated by the classical Mie theory ${ }^{44}$ based on the bulk dielectric function of $\mathrm{Au}^{45} \mathrm{D}$ is the NP diameter. $d_{0}$ is the path length of the spectrometer (usually about $1 \mathrm{~cm}$ ). Thus, based on the Eq. (2), $c$ (denoting the NP concentration) could be acquired. This concentration corresponded to the average NP-to-NP distance and could be adjusted by diluting the solution, which yield specific absorption coefficients (indicated by $\mu_{\mathrm{a}}$ ) ranging from $\sim 0.05$ to $\sim 1 \mathrm{~cm}^{-1}$ evaluated according to Beer-Lambert law. ${ }^{46}$ During the NP-laser interaction, the thermal field affected by a single NP was considered in the nanometer scale. ${ }^{47}$ The average distance between the NPs should be controlled with the order of magnitude of microns to avoid the thermal field coupling. In this work, even for NP suspensions with the highest concentration, the average particle spacing was estimated to be greater than several microns and thus the overlapping effect can be neglected.

The influence of the NP concentration $(c)$ was explored by changing the level of the absorption coefficient $\left(\mu_{\mathrm{a}}\right)$. Here, we used $\mu_{\mathrm{a}}$ instead of $c$ or extinction coefficient (which was considered proportional to $c$ ) to discuss the concentration influence on the photoacoustic effect of the medium evaporation because $\mu_{\mathrm{a}}$ is one of the important indicators of absorbed excitation energy, which is a crucial influencing factor of the photoacoustic signal induced by thermoelastic expansion. ${ }^{15}$

\section{Results and Discussion}

In this section, we present the photoacoustic responses to different operation conditions to investigate each key factor (e.g., excitation energy $E$, NP diameter $D$, and absorption coefficient $\mu_{\mathrm{a}}$ ) in sequence. The NP-mediated PTB dynamic characteristics function as a bridge for the analysis of photoacoustic response; hence, the relationship between the two is also explored. This section is organized as follows: Sec. 3.1 discusses the criterion of PTB generation. Section 3.2 provides a general description of the PTB-induced optical and acoustical responses. Sections 3.3, 3.4 , and 3.5 analyze in detail the photoacoustic responses to different factors.

\subsection{Criterion of Photothermal Bubbles Generation}

The bubble generation threshold energy $E_{\mathrm{th}}$ is considered crucial to improve the selectivity and efficacy of photothermal therapies and diagnostics. ${ }^{16-19}$ Although the method based on FDS is sensitive to bubble formation, the oscillation behavior cannot be observed easily when the excitation energy is close to the bubble threshold for small-sized NPs, because the FDS with short oscillation lifetime is usually undetectable or quenched somewhere by the presence of other NPs. ${ }^{41}$ Additionally, as the NP distribution in the suspension was an event with nondeterministic 


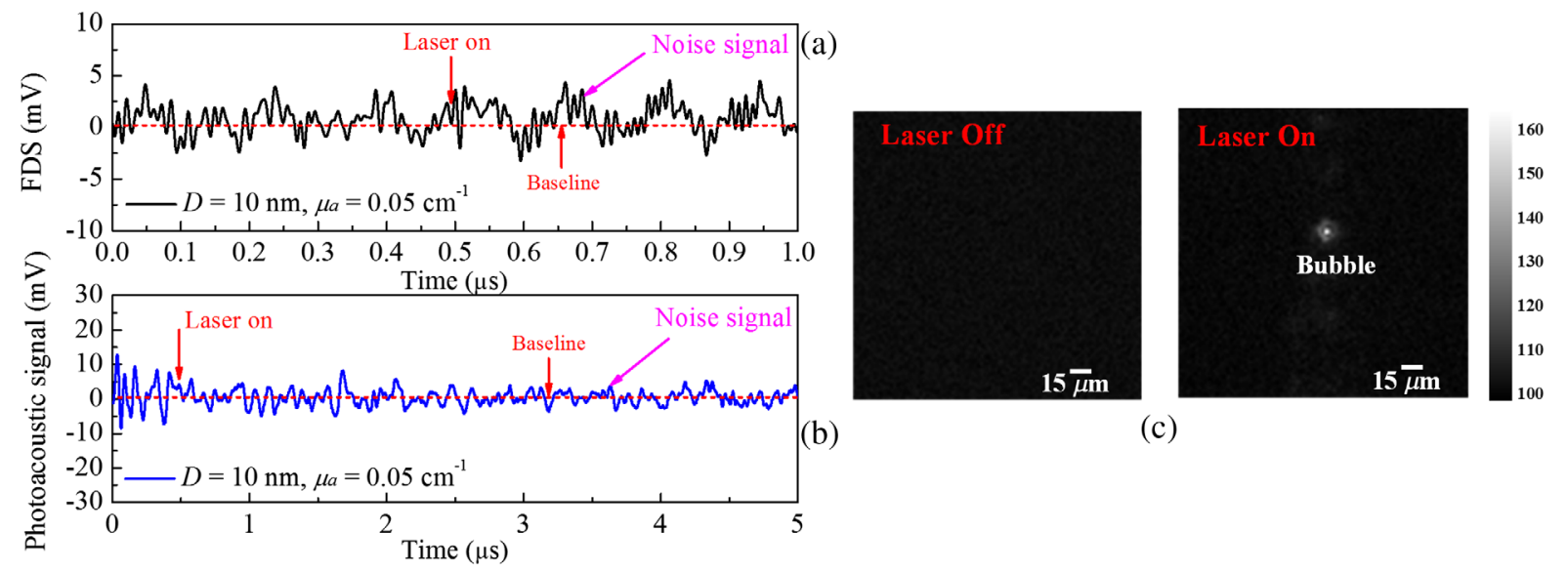

Fig. 3 Comparison of the multimodal detection of the bubble generation in the colloid gold NP suspensions, where the NP diameter and absorption coefficient are $10 \mathrm{~nm}$ and $0.05 \mathrm{~cm}^{-1}$, respectively. (a) FDS, (b) photoacoustic signal, and (c) side-scattering imaging with laser off (left) and on (right).

probability, bubble with misaligned probe beam usually linked with a several-fold decrease in the FDS amplitude, ${ }^{36}$ which further worsen the signal-to-noise contrast. Scattering imaging was the most sensitive, allowing us to observe any bubble generated in the EMCCD surface. As shown in Fig. 3, where 10-nm NP suspensions with $\mu_{\mathrm{a}}=0.05 \mathrm{~cm}^{-1}$ were irradiated with $E_{\mathrm{th}}$, the disturbance signal was so strong that it annihilated the photoacoustic signal and FDS. Thus, the side-scattering imaging technique could be used to assist the FDS method for a better assessment of $E_{\mathrm{th}}$. By contrast, although the method was the most sensitive, bubbles outside of the area or smaller than diffraction limit cannot be detected. Acoustic detection was advantageous for bubble identification in turbid media, ${ }^{48}$ but its sensitivity was limited to the transducer bandwidth. Thus, in summary, the criterion of bubble generation based on the three modalities can be described below: ${ }^{49}$ a positive bubble response can be confirmed once scattering image modality worked. If no bubble was captured in the image, but the time-resolved scattering and photoacoustic signals worked simultaneously, bubbles were also positively considered to be generated.
By applying the above criterion of identifying PTB generation, a series of experiments were conducted to extract the $E_{\mathrm{th}}$ of the suspensions with different NP sizes. A representative set of NPs $(D=10$ and $40 \mathrm{~nm})$ with $\mu_{\mathrm{a}}=0.05 \mathrm{~cm}^{-1}$ was chosen. Because there was a shot-to-shot fluctuation of excitation energy for the ns-pulsed laser illumination, to ensure a good reproducibility, only the experiment data with the pulse energy in the range of $\pm 5 \%$ for interested energy values were chosen. Hereinto, more than 50 pulses for each energy were excited to irradiate each sample over the range of radiant exposures and each bubble response was recorded correspondingly. Figure 4(a) shows the probability of bubble occurrence as a function of excitation energy. The profile of the bubble threshold could be well described by a sigmoid function, and the smaller of $D$, the higher of $E_{\mathrm{th}}$. The NP size effect of the bubble generation was further explored, as shown in Table 1, where the concentration was relatively high with $\mu_{\mathrm{a}}=1 \mathrm{~cm}^{-1}$. A significant increase in $E_{\mathrm{th}}$ with an increase in the NP diameter from 60 to $10 \mathrm{~nm}$ was observed, which can be attributed to (1) the fast energy release to the surroundings rather than being stored effectively in the interface for medium vaporization, and

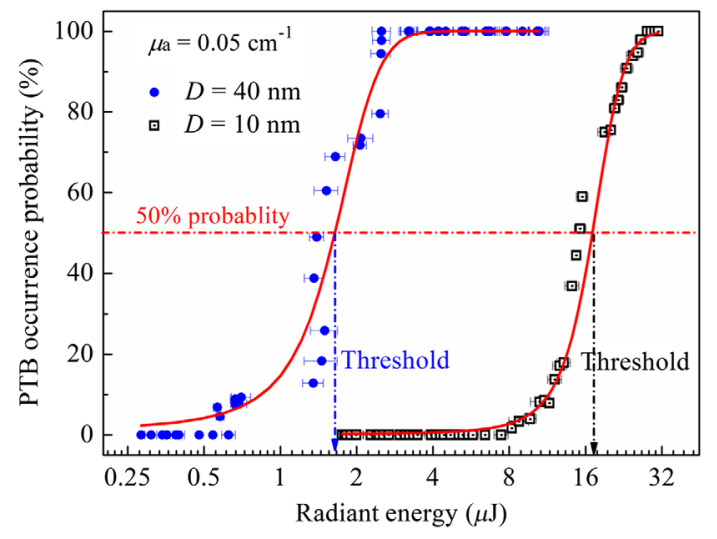

(a)

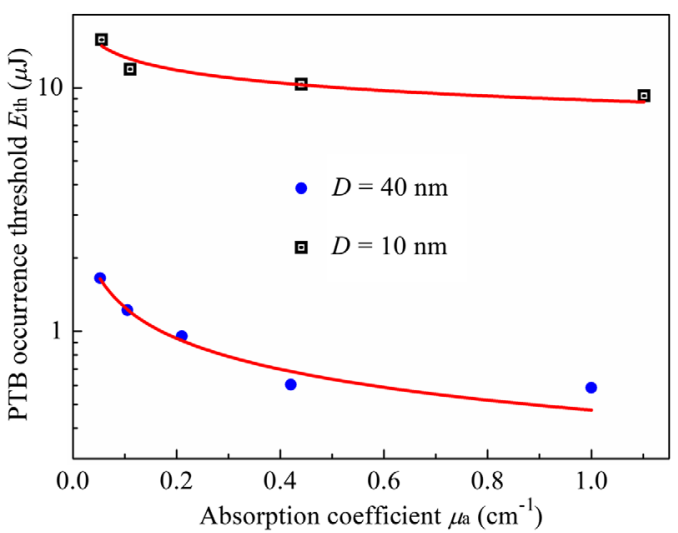

(b)

Fig. 4 Energy threshold for bubble generation. (a) The probability of PTB occurrence as a function of excitation energy. The diameters of the NPs in use are 10 and $40 \mathrm{~nm}$, respectively, with the absorption coefficient of $0.05 \mathrm{~cm}^{-1}$. The threshold probability line was defined as $50 \%$ probability for bubble occurrence. (b) Threshold as a function of the absorption coefficient. A polytropic exponential function can fit the experimental data well. The error bars indicated the standard deviation of the consecutive energy tracing at the preset $E$. 
Table 1 NP size effect on PTB threshold with $\mu_{\mathrm{a}}$ approximating to $1 \mathrm{~cm}^{-1}$.

\begin{tabular}{lcccccc}
$D(\mathrm{~nm})$ & 60 & 50 & 40 & 20 & 15 & 10 \\
\hline$\mu_{\mathrm{a}}\left(\mathrm{cm}^{-1}\right)$ & 0.93 & 1.01 & 1.05 & 1.08 & 1.09 & 1.1 \\
$E_{\text {th }}(\mu \mathrm{J})$ & $0.436 \pm 0.039$ & $0.483 \pm 0.060$ & $0.586 \pm 0.049$ & $3.170 \pm 0.429$ & $3.562 \pm 0.419$ & $9.274 \pm 1.288$ \\
\hline
\end{tabular}

(2) the inefficient laser deposition due to low absorption properties, for small-sized NP.

In fact, the ensemble effect of NPs in the suspension, not a single NP, was our concern, and the $E_{\text {th }}$ was influenced radically by not only NP size but also the agglomeration (concentration). As shown in Fig. 4(b), $E_{\text {th }}$ increases with the decrease of the $\mu_{\mathrm{a}}$. For each NP, $E_{\text {th }}$ was proportional to $\mu_{\mathrm{a}}^{\gamma}$, where $\gamma$ was $\sim-1 / 3$. For the $40-\mathrm{nm}$ NP suspensions, $E_{\mathrm{th}}$ was $0.59 \mu \mathrm{J}$ for $\mu_{\mathrm{a}}=$ $1 \mathrm{~cm}^{-1}$ and $1.65 \mu \mathrm{J}$ for $\mu_{\mathrm{a}}=0.05 \mathrm{~cm}^{-1}$. For the 10-nm NP case, $E_{\mathrm{th}}=9.27 \mu \mathrm{J}$ when $\mu_{\mathrm{a}}=1.10 \mathrm{~cm}^{-1}$ and $E_{\mathrm{th}}=15.75 \mu \mathrm{J}$ when $\mu_{\mathrm{a}}=0.05 \mathrm{~cm}^{-1}$. The influence of the NP concentration can be explained below. The bubble was expected to originally appear in the focal point because of the highest laser intensity, whereas, in practice, as the generation of PTB occurs at the location of the NP, the $E_{\text {th }}$ of PTB will be also dominated by the NP distribution. In our experiments, a small absorption coefficient usually indicates low NP concentration and large NP-to-NP space, which will reduce the frequency of statistical events, i.e., the PTB occurrence, thereby yielding an overestimated $E_{\text {th }}$ [see Fig. 4(b)]. By contrast, the suspensions with relatively high absorption coefficient are expected to approximate the case of individual NP-laser interaction because of a compact spatial distributive characteristic.

The phase transition caused by the thermal accumulation, which is localized in the medium around the NP, is a mechanism to improve the traditional photothermal therapy. ${ }^{11}$ However, the photothermal response of an isolated NP cannot be explored directly, although the isolated NP assumption is considered the best model in studying the photothermal effect. Moreover, because of the optical diffraction limit, optical microscopy cannot easily exhibit the image of the nanosized dimension. ${ }^{36}$ The optical scattering technique based on bubble scattering can improve the optical identification of the bubble generation. However, the fixed trestle of NPs, such as glass substrate, increased the bubble curvature and resulted in a significant increase in the surface tension, and thus the bubble formation in the substrate-bound case was far more difficult than that of a free suspended NP in solution. ${ }^{50,51}$ As a summary, because geometry restriction and the single-mode detection method resulted in improper estimations of the threshold, the multimodal coupling detection in this paper can reasonably estimate the threshold energy through an ensemble study using the NP suspensions.

\subsection{Optical and Acoustic Responses of Nanoparticle-Mediated Photothermal Bubbles}

To characterize the optical and acoustic signals induced by PTB, three different levels of excitation energy were chosen to irradiate the 50-nm NP suspension with $\mu_{\mathrm{a}}=1 \mathrm{~cm}^{-1}$. As shown in Fig. 5, with an increase in the radiant energy from 3.86 to $50 \mu \mathrm{J}$, a U-shaped [Fig. 5(a)] and a V-shaped [in Figs. 5(b) and 5(c)] bubble-specific FDS appeared successively. The valley width of the FDS curve indicated oscillation time $T_{\text {osc }}$, which approximately characterized the bubble lifetime from expansion to collapse. $T_{\text {osc }}$ increased with $E$, as shown in Figs. 5(a)-5(c). After laser emission, a polar transient wave at a specific delay time of $\sim 2.8 \mu$ s was observed, whose peak magnitude was defined as photoacoustic signal amplitude (denoted as $P$ ). The delay time characterized the propagation time of acoustic wave from the bubble generation location to the active area of the ultrasound transducer; thus, it was almost constant under different excitations but depended on the experimental setup.

Two distinctive phenomena were noted on the time-resolved response profiles. On the one hand, a very sharp V-profile FDS could be observed at the initial stage of the optical response [see Figs. 5(b) and 5(c)] when the excitation energy $E$ was far beyond $E_{\mathrm{th}}$. Under such high excitation energy, the transient high temperature could aggrandize the thermal stress wave and alter the refractive index of the adjacent liquid, which finally led to the saltatorial light deflection in a very short time scale. On the other hand, a second remarkable photoacoustic wave with further augmentation of the irradiation energy was observed, as shown in Fig. 5(c). The bubble oscillation period $(1.4 \mu \mathrm{s})$ was found to be approximately equal to the time interval between the peaks of the two adjacent photoacoustic signals, which corresponded to the rapid movements of the bubble wall during expansion and collapse stages.

We further investigated the relationship between the photoacoustic signal amplitude $P$ and the bubble oscillation time up to $T_{\text {osc }}=0.8 \mu \mathrm{s}$ in Fig. 6, where an almost linear correlation between the two quantities was shown. Therefore, the photoacoustic signal stemmed mainly from the bubble dynamics. When the energy was relatively low, then the acoustic signal produced by bubble collapse became indistinct because it was too weak to be detected by the transducer due to the damping of bubble kinetics by water viscosity and bubble-water interfacial evaporation-condensation process.

\subsection{Effects of Excitation Energy}

During the NP-mediated bubble generation excited by an intense pulsed laser, a rapid and dynamic modification of the absorption properties, the NP intrinsic shape and structure, ${ }^{42}$ and the thermophysical parameters (thermal expansion coefficient, density, speed of sound, and heat capacity) of NP-water microsystem ${ }^{17}$ had been reported. The real situation involving bubble population in the suspension was even complex. Existing theoretical studies based on the assumption of ideal condition with constant parameters have difficulty in revealing the physical mechanisms behind the phenomenon. In fact, in the real applications of NP-based therapies, the ensemble state of NP in the suspensions is a typical NP state that requires attention. To dissect the complex relationship of the physical quantities (i.e., $P$ corresponding to diagnostic effect and $R_{\max }$ during time evolution associated with therapy quality) involved in a theranostics system, this section characterizes quantitatively 


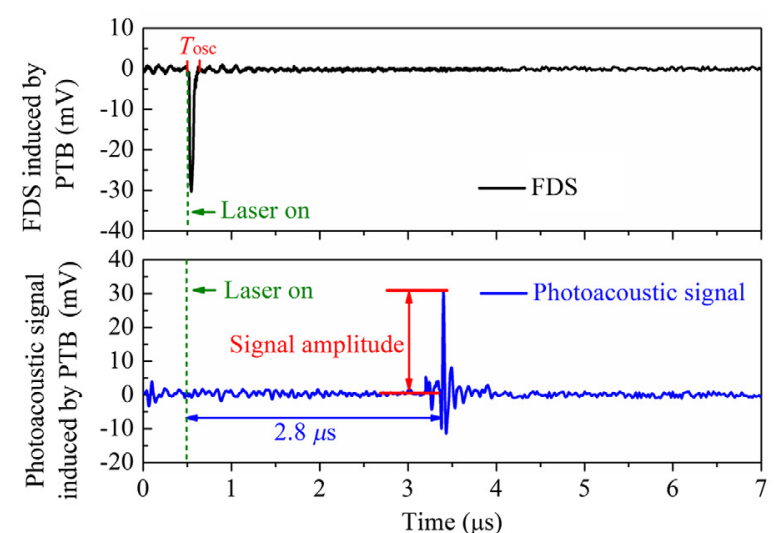

(a)

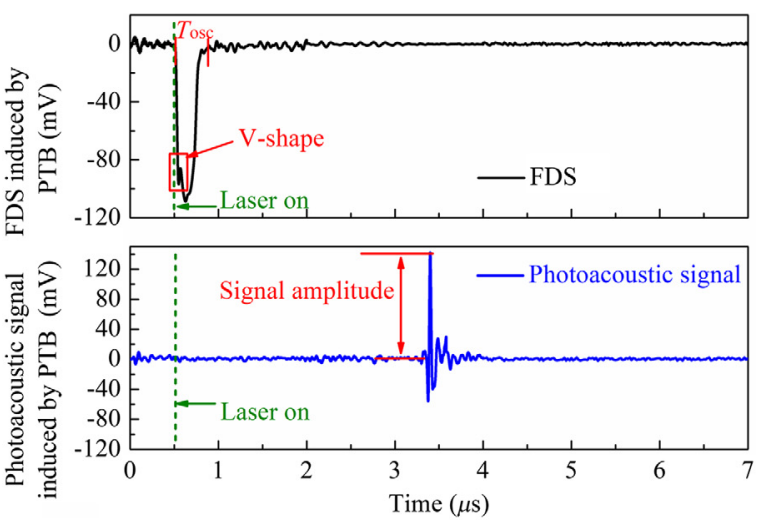

(b)
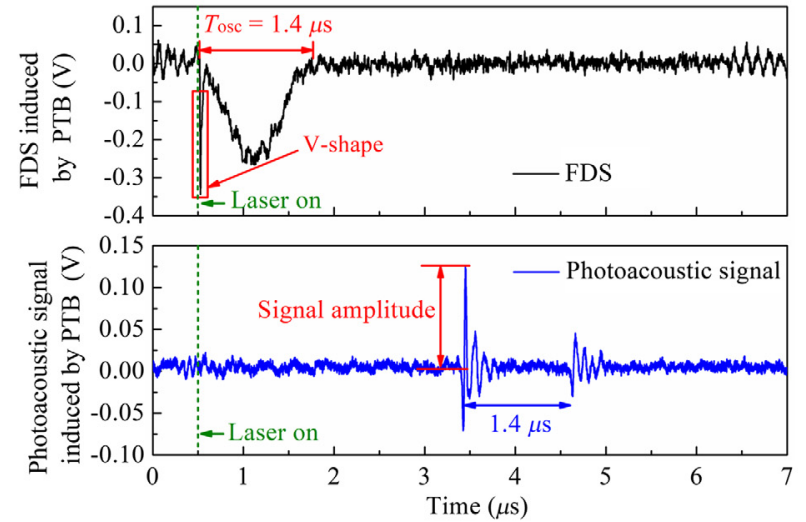

(c)

Fig. 5 Typical time-resolved photoacoustic and FDS signals induced by PTB in the 50 -nm NP suspension with an absorption coefficient of $\sim 1 \mathrm{~cm}^{-1}$ under different laser excitations: (a) 3.86 , (b) 27.8 , and (c) $50 \mu \mathrm{J}$. The green dashed line denotes the trigger of excitation laser.

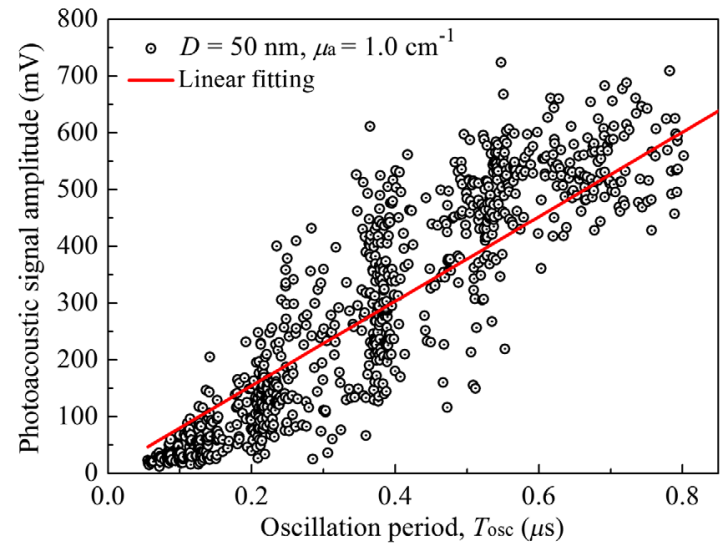

Fig. 6 Quantitative expression of the measured acoustic signal (photoacoustic signal amplitude) with regard to the optical response (the bubble oscillation period).

the signal responses to the regulation parameter of the incident energy $E$ in NP suspensions.

Two types of NP suspensions were chosen: 50-nm NP suspension with $\mu_{\mathrm{a}}=0.37 \mathrm{~cm}^{-1}$ and 15-nm NP suspension with $\mu_{\mathrm{a}}=1.09 \mathrm{~cm}^{-1}$. The suspensions were irradiated with excitation energies ranging from the $E_{\text {th }}$ to 1.2 folds the upper limit of the optical breakdown threshold energy $E_{\mathrm{CBO}}=250 \mu \mathrm{J}$ in the NP-free solutions. The maximum bubble size $R_{\max }$ can be deduced approximately based on Eq. (1), and hence, we showed the relationship between $R_{\max }$ and $E$ in Fig. 7, where a generalized decaying exponential function of $\exp (-\alpha E)$ ( $\alpha$ is the fitting constant) could be used to describe the measured results. The profiles of the photoacoustic signal amplitude $P$ with regard to $E$ are totally distinct: For the 15-nm NP suspensions with a high absorption coefficient $\left(\mu_{\mathrm{a}}=1.09 \mathrm{~cm}^{-1}\right)$, a linear correlation between $P$ and $E$ was found [Fig. 7(b)]. Moreover, in the 50-nm case, the correlation deviated significantly from the linear representation when $E>80 \mu \mathrm{J}$, as shown in Fig. 7(d). It can be summarized that with a low level of excitation energy, the signal responses can be considered a linear correlation to the excitation energy, regardless of the NP diameter and concentration. Hence, it was not difficult to find a linear curve to well describe the relation between $P$ (or $R_{\max }$ ) and $E$ within the range, for example, from $E_{\mathrm{th}}$ to 20 folds greater. Within this energy range, a fairly linear relationship had been experimentally demonstrated efficient, as shown in Figs. 7(e) and 7(f), respectively. The original linear representation in the low-energy range was taken as the reference to evaluate the signal trend with high energy. When $90 \%$ of the experimental points started to deviate from the above original linear fitting curve, we defined the critical excitation energy as the cutoff point $E_{\text {linear }}$, which was the turning point of $P$ from the enhancement characteristic region to the saturation characteristic region. Inspection of Figs. 7(a) and 7(c), when stretched to a high level of excitation energy, the bubble 


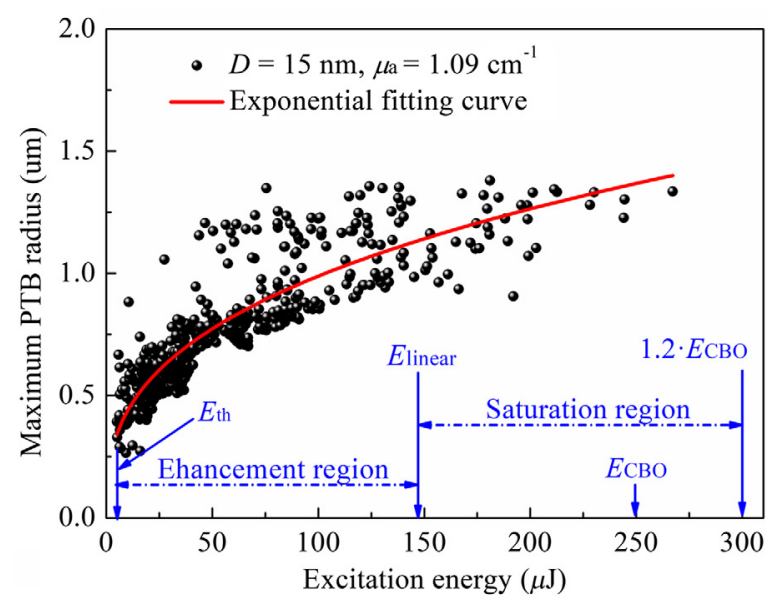

(a)

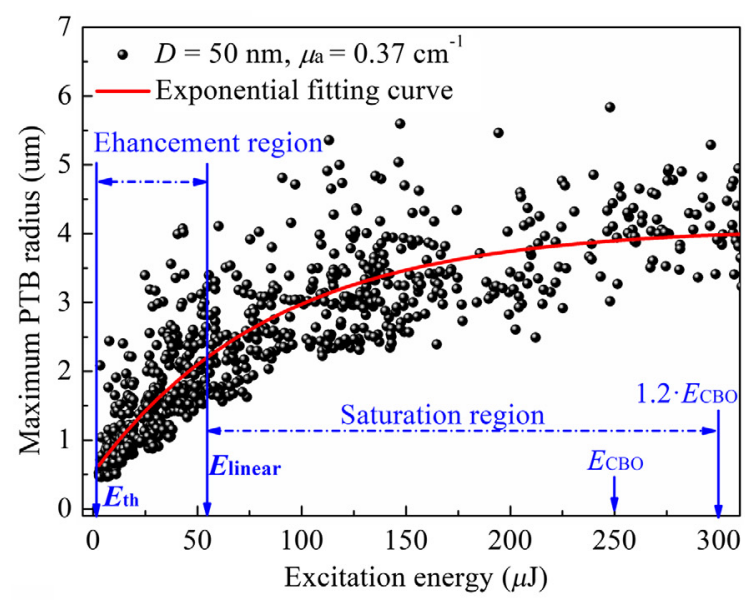

(c)

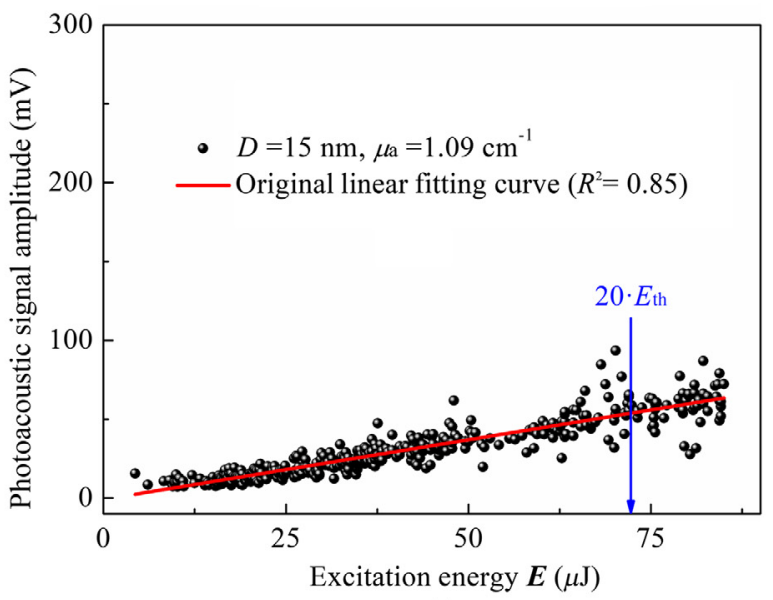

(e)

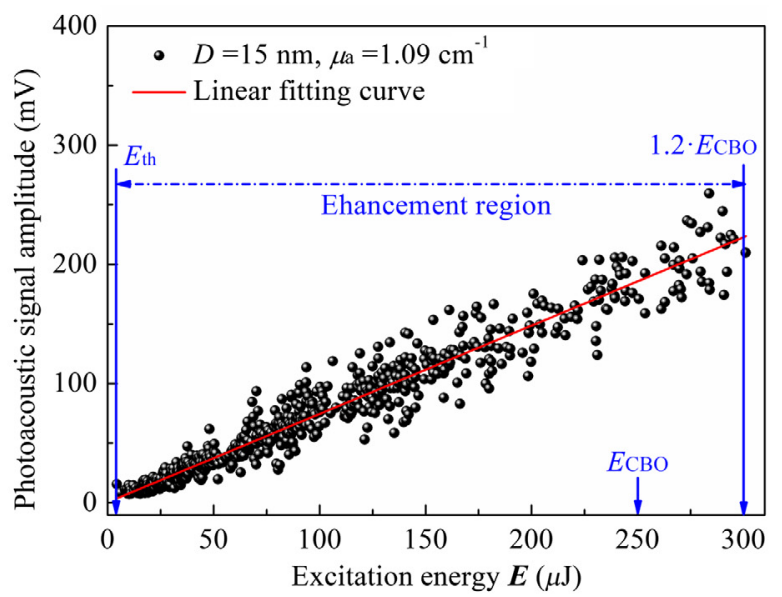

(b)

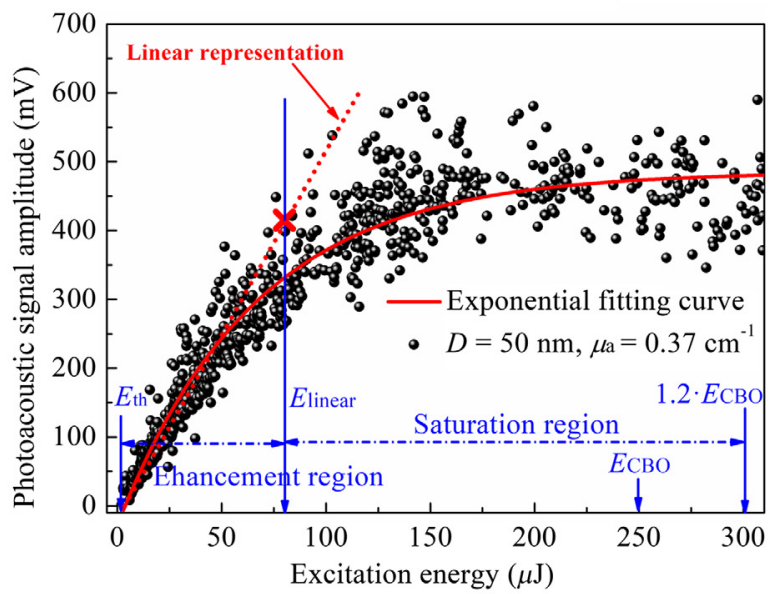

(d)

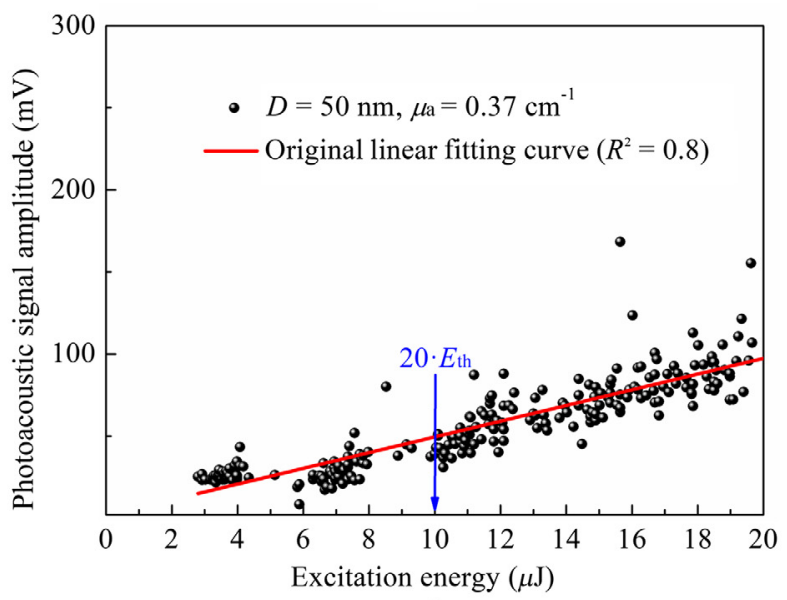

(f)

Fig. 7 Comparison of photoacoustic signal and PTB size stemming from FDS as a function of excitation energy. (a) and (b) Denoted the 15-nm NP suspensions with an absorption coefficient of $1.09 \mathrm{~cm}^{-1}$. (c) and (d) Denoted the 50-nm NP suspensions with an absorption coefficient of $0.37 \mathrm{~cm}^{-1}$. (e) and (f) Illustrated the original linear representation curve. $E_{\text {linear }}$, indicates the termination of the linear energy range and $E_{\mathrm{CBO}}$ indicates the threshold energy of optical breakdown in the NP-free solutions. Exponential and linear functions were used to fit the acquired experimental signals.

size saturation occurred, whereas photoacoustic behavior exhibited different representations [see Figs. 7(b) and 7(d)]. In the enhancement region, signal amplitude was in direct proportion to $E$, which meant a relatively high and stable energy conversion efficiency from the light energy to acoustic or bubble kinetics. In the saturation region, the conversion efficiency was inhibited and declined gradually with the excitation energy. 

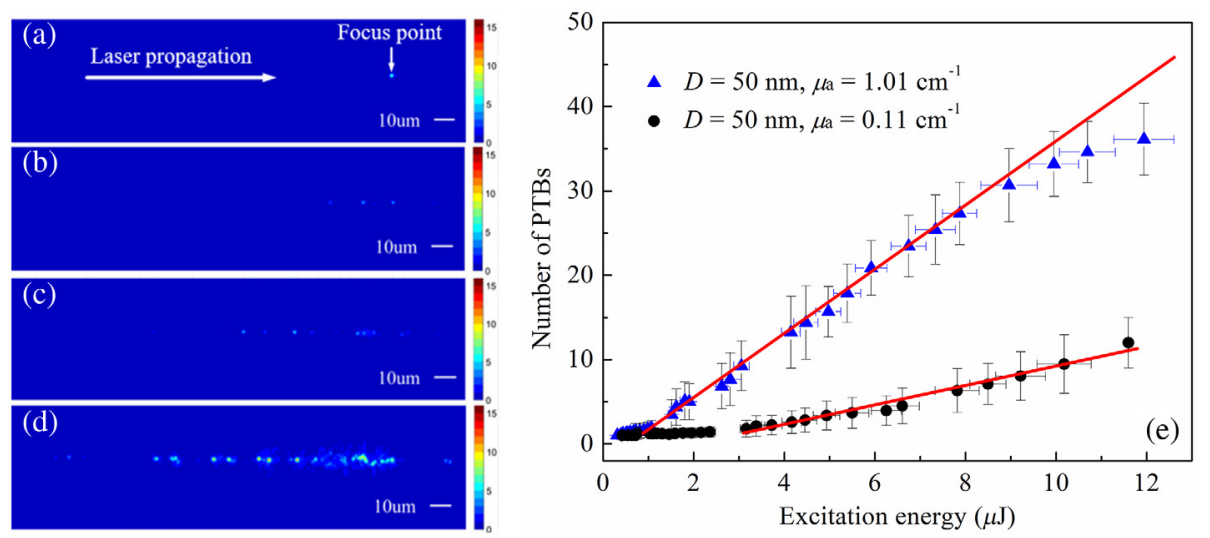

Fig. 8 PTB sites versus excitation energy based on the side-scattering imaging method. The acquired SDS was presented through the pseudo-color processing. About 50-nm gold NP suspensions with $\mu_{\mathrm{a}}=$ $1 \mathrm{~cm}^{-1}$ were irradiated by excitation energy of (a) 0.69 , (b) 0.95 , (c) 1.78 , and (d) $4.33 \mu \mathrm{J}$. The horizontal arrow denotes the laser propagation direction. The beam focus is marked by the vertical arrow. The color bar is the normalized side-scattering light intensity. (e) The quantity of PTB sites along the excitation energy at different absorption coefficients.

The first issue is to elucidate the appearance of saturation characteristic region, which can be attributed to three aspects. First, with the increasing of $E$, the thermal phase transition of NP might happen, ${ }^{52}$ which will suppress the heat transfer to the surroundings, yielding a low energy conversion efficiency. Second, the PTB formation can limit the NP optical absorption but enhance the scattering and finally bleach the localized plasmon resonance band, which will reduce further the conversion efficiency. ${ }^{53}$ Third, the occurrence of PTB population will result in the shielding effect, which was demonstrated in detail below (Fig. 8). To throw some light on the phenomenon of population shielding effect, side-scattering imaging was used to excavate the spatial quantitative information of PTBs. A quantitative criterion of bubble generation in scattering image had been detailed in Ref. 28. Figures 8(a)-8(d) shows the spatial distribution of PTB sites, where 50-nm NP suspension with $\mu_{\mathrm{a}}=1.0 \mathrm{~cm}^{-1}$ was taken as the irradiated sample. The grayscale imaging acquired by the EMCCD was processed with pseudocolors to improve the visualization. The bright spots represented the PTB occurrence sites, which increased along with the excitation energy from $0.69 \mu \mathrm{J}$ (close to $E_{\text {th }}$ ) to $4.33 \mu \mathrm{J}$ (about 6 times of $\left.E_{\text {th }}\right)$. Hereinto, PTB first formed in a small region around the beam waist. As the radiant energy increased, the PTB threshold might be met at distances far away from the focal point. Thus, the PTB initiation sites moved along the beam axis, and as a result, more bubbles occurred. A quantitative estimation of the PTB sites along the optical axis is shown in Fig. 8(e). The target energy level was restricted to have a deviation $<5 \%$ of the nominal value due to the shot-to-shot energy fluctuation of ns pulsed laser. At least 50 experimental images for each case were conducted and analyzed to ensure good reproducibility. Results showed that the measured data had a slight deviation from the linear fitting curve for the 50-nm NP suspension with $\mu_{\mathrm{a}}=1 \mathrm{~cm}^{-1}$. By contrast, less PTB sites with low NP concentration showed up in a fairly linear way, where $\mu_{\mathrm{a}}=0.11 \mathrm{~cm}^{-1}$. The extinction of the laser energy by the PTB population formation will cause a serious decrease in the amount of energy available to produce new PTBs, which was called the PTB shielding effect. This effect became prominent with the increase of $D$ and $c$ because large NPs and high absorption coefficients always indicated a high possibility of large and numerous PTBs.

The second issue is the quantification of the parameter relationship involved in a theranostics system. First, the NP size effect on the cutoff points $E_{\text {linear }}$ of the bubble size and bubble dynamic-induced photoacoustic responses (to excitation energy) is studied and shown in Table 2, where $\mu_{\mathrm{a}}$ approximated to $1 \mathrm{~cm}^{-1}$. The results showed both signal responses will turn into the saturation region more easily for large NPs and the saturation region will vanish if the NP diameter was small enough. For example, saturated acoustic signals cannot be observed with the 15- and 10-nm NP suspensions, respectively. The ratio of $E_{\text {linear }}$ corresponding to two signal responses was calculated, and the latter (for acoustic response) was found $\sim 1.2$ to 1.3 times greater than the former (for optics-based bubble size) for the same NP size. This finding implied that a linear dependence existed between the two signals, but it did not always remain in effect. In addition, the cutoff value of $E_{\text {linear }}$ normalized by $E_{\text {th }}$ for characterizing the linear response to the incident energy was also evaluated, where $E_{\mathrm{th}}$ could be found in Table 1. Results showed that the level of the normalized cutoff value dispersed heavily with respect to the NP size. In this work, we provided a recommended parameter, which can describe the linear relationship of the signal response for all NP sizes. This parameter was typically the minimum normalized value of $E_{\text {linear }} / E_{\mathrm{th}}$. In summary, 40 folds of $E_{\text {th }}$ were recommended for the linear response for bubble size and 57 folds of $E_{\mathrm{th}}$ were recommended for photoacoustic signal. This finding indicated the linear relationship between the photoacoustic signal and the (maximum) PTB size existed only in a confined energy range from $E_{\mathrm{th}}$ to $\sim 40$-fold larger regardless of the NP size, beyond which it was complicated to construct the dependence curve.

\subsection{Nanoparticle Size Effect}

In this section, NPs with diameters ranging from 10 to $60 \mathrm{~nm}$ were adopted and the corresponding suspensions were excited by $E=15$ and $200 \mu \mathrm{J}$, which were affiliated with the photoacoustic enhancement and saturation region (if it existed), respectively. To ensure a high contrast of signal-to-noise at 
Table $2 E_{\text {linear }}$ of the photoacoustic signal and bubble size for different NP sizes with a high level $\mu_{\mathrm{a}}\left(\sim 1 \mathrm{~cm}^{-1}\right)$.

\begin{tabular}{lcccccc}
$D(\mathrm{~nm})$ & 60 & 50 & 40 & 20 & 15 & 10 \\
\hline$\mu_{\mathrm{a}}\left(\mathrm{cm}^{-1}\right)$ & 0.93 & 1.01 & 1.05 & 1.08 & 1.09 & 1.1 \\
$E_{\text {linear }}$ of photoacoustic signal $(\mu \mathrm{J})$ & 50 & 61.5 & 103 & 180 & - & - \\
$E_{\text {linear }} / E_{\text {th }}$ for photoacoustic signal & 115 & 127 & 176 & 57 & - & - \\
$E_{\text {linear }}$ of bubble size $(\mu \mathrm{J})$ & 38 & 50 & 82 & 138 & 145 & - \\
$E_{\text {linear }} / E_{\text {th }}$ for bubble size & 87 & 104 & 140 & 44 & 40 & -
\end{tabular}

a low energy, suspensions with high NP concentration (where $\mu_{\mathrm{a}}$ was $\sim 1 \mathrm{~cm}^{-1}$ ) were chosen.

As shown in Fig. 9, the PTB-induced photoacoustic amplitude $P$ was normalized by the excitation energy $E$ and the normalized value sharply increased with particle size regardless of excitation (enhancement and saturation) region. This situation is entirely different from the photoacoustic signals triggered by the mechanism of thermal expansion, ${ }^{11,15}$ where the photoacoustic amplitude is not sensitive to the NP size with the identical absorption coefficient. The normalized value under the photoacoustic enhancement regime $(15 \mu \mathrm{J})$ was prominently higher than that of photoacoustic saturation regime $(200 \mu \mathrm{J})$. A polytropic exponential function could be used to well describe the corresponding curves where the fitting exponents were $\sim 1.82$ and 1.2 for the enhancement and saturation, respectively. The results indicated that high excitation energy can possibly overshadow the NP size effect on the photoacoustic signal. Thus, the critical threshold of $E_{\text {linear }}$ (which had been summarized in Sec. 3.3) was crucial to distinguish the response regions for an efficient utilization of NP size effect in the biosensing field. Hereinto the quantification of labeled biomolecules depended on NP agglomeration degree, ${ }^{9,26}$ which can be understood as a particle size changing process. Thus, the PTB-based photoacoustic system may be more sensitive than other conventional spectroscopy methods. Hereinto, the effect considered here could increase the acoustical signal amplitude without a significant change in the absorption coefficient.

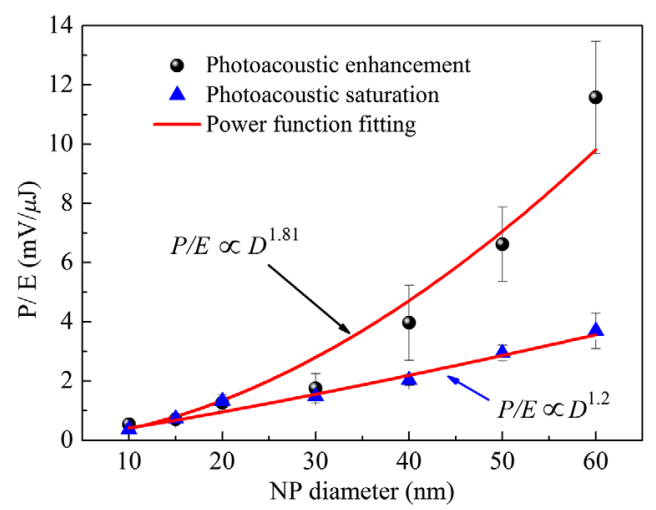

Fig. 9 Normalized (by the excitation energy) photoacoustic signal as a function of NP size irradiated by an excitation energy of 15 (in the enhancement regime) and $200 \mu \mathrm{J}$ (in the saturation regime).

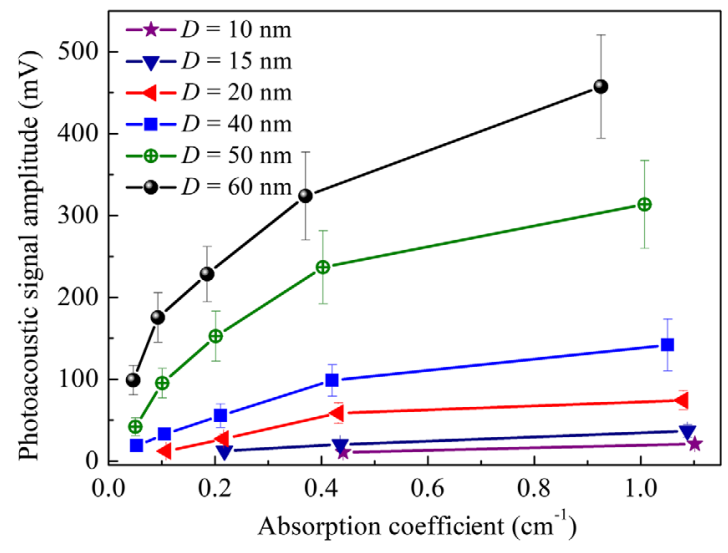

Fig. 10 Effects of absorption coefficient $\mu_{\mathrm{a}}$ (i.e., the NP concentration) on the photoacoustic signal amplitude.

\subsection{Effect of Nanoparticle Concentration}

NP suspensions (10 to $60 \mathrm{~nm}$ in diameter) with well-tuned absorption coefficients from 0.05 to $1.1 \mathrm{~cm}^{-1}$ were prepared. As shown in Fig. 10, the photoacoustic signal amplitude $P$ showed a monotonically increasing behavior with $\mu_{\mathrm{a}}$ and $D$ but exhibited different increasing rates along the absorption coefficient for various NPs. The relationship between $P$ and $\mu_{\mathrm{a}}$ for small NPs (with $D$ below $20 \mathrm{~nm}$ ) can be linearly approximated. Moreover, with an increase in the NP size, the fitting power exponent parameter $n(<1$ in the exponential function expressed by $\mu_{\mathrm{a}}^{n}$ ) described the nonlinear growth of the amplitude along the absorption coefficient better than that of a linear fitting curve.

Detailly, although high NP concentration will result in the large amplification of photoacoustic amplitude due to the rapid PTB proliferation [see Fig. 8(e)], the argument that "the higher, the better" is not always true. A 2.5 -fold increase in concentration of the $60-\mathrm{nm} \mathrm{NP}$ suspension from $\mu_{\mathrm{a}}=0.37 \mathrm{~cm}^{-1}$ to

Table 3 Summary of influences of different factors on photoacoustic signal response.

Experimental parameter Photoacoustic signal amplitude $P$

Excitation energy

(E)

NP concentration (indicated by $\mu_{\mathrm{a}}$ )

Maximum bubble size $\left(R_{\max }\right)$ 
$\mu_{\mathrm{a}}=0.93 \mathrm{~cm}^{-1}$ only provide a 1.4 -fold enhancement of photoacoustic amplitude. The enhancement is expected to further weaken with an increase in the suspension concentration. The 60-nm NP had the lowest threshold (see Table 1) due to the maximum heating efficiency. Thus, more bubbles will be generated and thus higher signal amplitude can be observed, compared with that of small-sized NP with the identical absorption coefficients as shown in Fig. 8. However, the rapid bubble proliferation with high absorption coefficient will easily suffer the PTB shielding, which in turn will suppress the proliferation rate (see Fig. 8). Therefore, a decreased increasing rate of the photoacoustic signal with the increase of $\mu_{\mathrm{a}}$ is shown in Fig. 10 and summarized in Table 3, where the impacts of different factors had been summarized in a table.

\section{Conclusion}

We developed a proof-of-principle multimodal platform to systematically study the photoacoustic signal induced by the laserexcited PTBs in colloidal gold NP suspensions. The results show that compared with the bubble generation threshold, a conservative estimation of the critical level is $\sim 40$ folds higher, below which the photoacoustic signal exhibits a linear dependence on the PTB size. The photoacoustic signal amplitude depends strongly on excitation energy, NP size, and concentration. The signal amplitude exhibits a multistage behavior with regard to the excitation energy, which can be divided into two regions: the enhancement characteristic region, where the signal linearly increased with the excitation energy and can be scaled quadratically by NP size, and the saturation characteristic region, where the signal amplification with regard to the excitation energy is impeded and is in direct proportion to the NP size. As for the effects of NP concentration, a higher photoacoustic signal amplitude is generally induced by a dense NP distribution, but this condition is not always valid, especially in the case of large particles, where the shielding effect of the NP swarm prevents the increase in the photoacoustic response.

This work studied systematically the photoacoustic signal responses of multiscale bubble sizes triggered by excitation conditions with multiple factors. The work can help construct the theranostics model to realize real-time dose adjustments by monitoring the photoacoustic signal. In particular, the findings could be advanced for the implementation of theranostics in the clinical applications from nanoscale gene therapies, nanoor microscale tumor margin resection, to microscale vascular applications.

\section{Disclosures}

The authors have no relevant financial interests in this article and no potential conflicts of interest to disclose.

\section{Acknowledgments}

This work was supported jointly by the National Natural Science Foundation (Grant Nos. 61335012, 61727823, 61575156, 61705177, 61775178, and 61505159).

\section{References}

1. J. P. M. Almeida et al., "In vivo gold nanoparticle delivery of peptide vaccine induces anti-tumor immune response in prophylactic and therapeutic tumor models," Small 11(12), 1453-1459 (2015).

2. S. Eustis et al., "Why gold nanoparticles are more precious than pretty gold: noble metal surface plasmon resonance and its enhancement of the radiative and nonradiative properties of nanocrystals of different shapes," Chem. Soc. Rev. 35(3), 209-217 (2006).

3. M. Hu et al., "Gold nanostructures: engineering their plasmonic properties for biomedical applications," Chem. Soc. Rev. 35(11), 1084-1094 (2006).

4. R. Shukla et al., "Biocompatibility of gold nanoparticles and their endocytotic fate inside the cellular compartment: a microscopic overview," Langmuir 21(23), 10644-10654 (2005).

5. J. J. Yao et al., "High-speed label-free functional photoacoustic microscopy of mouse brain in action," Nat. Methods. 12(5), 407-410 (2015).

6. L. D. Wang et al., "Grueneisen relaxation photoacoustic microscopy," Phys. Rev. Lett. 113(17), 174301 (2014).

7. C. L. Bayer et al., "Photoacoustic signal amplification through plasmonic nanoparticle aggregation," J. Biomed. Opt. 18(1), 016001 (2013).

8. H. Y. Ju et al., "Gold nanoparticle targeted photoacoustic cavitation for potential deep tissue imaging and therapy," Biomed. Opt. Express 4(1), 66-76 (2013).

9. X. J. Liu et al., "Strong size-dependent photoacoustic effect on gold nanoparticles: a sensitive tool for aggregation-based colorimetric protein detection," Anal. Methods 4(1), 309-311 (2012).

10. A. Buchkremer et al., "Enhanced photoacoustic signal from DNA assembled gold nanoparticle networks," Mater. Res. Express 1(4), 045015 (2014).

11. K. Wilson et al., "Biomedical photoacoustics beyond thermal expansion using triggered nanodroplet vaporization for contrast-enhanced imaging," Nat. Commun. 3, 618 (2012).

12. S. H. Yang, F. Ye, and D. Xing, "Intracellular label-free gold nanorods imaging with photoacoustic microscopy," Opt. Express 20(9), 1037010375 (2012).

13. F. Gao et al., "Rationally encapsulated gold nanorods improving both linear and nonlinear photoacoustic imaging contrast in vivo," Nanoscale 9(1), 79-86 (2017).

14. A. Prost et al., "Photoacoustic generation by a gold nanosphere: from linear to nonlinear thermoelastics in the long-pulse illumination regime," Phys. Rev. B 92(11), 115450 (2015).

15. G. A. Pang et al., "Photoacoustic signal generation in gold nanospheres in aqueous solution: signal generation enhancement and particle diameter effects," J. Phys. Chem. C 120(48), 27646-27656 (2016).

16. F. Gao et al., "Single laser pulse generates dual photoacoustic signals for differential contrast photoacoustic imaging," Sci. Rep. 7(1), 626 (2017).

17. S. Y. Nam et al., "Nonlinear photoacoustic signal increase from endocytosis of gold nanoparticles," Opt. Lett. 37(22), 4708-4710 (2012).

18. Y. S. Chen et al., "Silica-coated gold nanorods as photoacoustic signal nanoamplifiers," Nano Lett. 11(2), 348-354 (2011).

19. M. W. Sigrist et al., "Laser generation of acoustic waves in liquids and gases," J. Appl. Phys. 60(7), R83-R122 (1986).

20. E. Y. Lukianova-Hleb et al., "Intraoperative diagnostics and elimination of residual microtumours with plasmonic nanobubbles," Nat. Nanotechnol. 11(6), 525-532 (2016).

21. E. Y. Lukianova-Hleb et al., "Tunable plasmonic nanobubbles for cell theranostics," Nanotechnology 21(8), 85102 (2010).

22. J. Zhao et al., "Cancer theranostics with gold nanoshells," Nanomedicine 9(13), 2041-2057 (2014).

23. M. Hu et al., "Investigation of the properties of gold nanoparticles in aqueous solution at extremely high lattice temperatures," Chem. Phys. Lett. 391(4-6), 220-225 (2004).

24. V. Kotaidis et al., "Excitation of nanoscale vapor bubbles at the surface of gold nanoparticles in water," J. Chem. Phys. 124(18), 184702 (2006).

25. V. P. Zharov, "Ultrasharp nonlinear photothermal and photoacoustic resonances and holes beyond the spectral limit," Nat. Photonics 5(2), 110-116 (2011).

26. M. G. Gonzalez et al., "Strong size-dependent photoacoustic effect on gold nanoparticles by laser-induced nanobubbles," Appl. Phys. Lett. 96(17), 174104 (2010).

27. C. P. Lin et al., "Cavitation and acoustic emission around laser-heated microparticles," Appl. Phys. Lett. 72(22), 2800-2802 (1998).

28. D. Lapotko, "Plasmonic nanoparticle-generated photothermal bubbles and their biomedical applications," Nanomedicine 4(7), 813-845 (2009).

29. R. H. Xiong et al., "Comparison of gold nanoparticle mediated photoporation: vapor nanobubbles outperform direct heating for delivering macromolecules in live cells," ACS Nano 8(6), 6288-6296 (2014). 
30. L. Tong et al., "Gold nanorods mediate tumor cell death by compromising membrane integrity," Adv. Mater. 19(20), 3136-3141 (2007).

31. C. Ungureanu et al., "Light interactions with gold nanorods and cells: implications for photothermal nanotherapeutics," Nano Lett. 11(5), 1887-1894 (2011).

32. M. Kitz et al., "Vapor bubble generation around gold nanoparticles and its application to damaging of cells," Biomed. Opt. Express 2(2), 291304 (2011).

33. Y. Feng et al., "Occlusion and rupture of ex vivo capillary bifurcation due to acoustic droplet vaporization," Appl. Phys. Lett. 112(23), 233701 (2018).

34. A. Sengupta et al., "Delivery of siRNA to ovarian cancer cells using laser-activated carbon nanoparticles," Nanomedicine 10(11), 17751784 (2015).

35. K. Kawabata et al., "Acoustic response of microbubbles derived from phase-change nanodroplet," Jpn. J. Appl. Phys. 49(7), 07HF18 (2010).

36. E. Y. Lukianova-Hleb et al., "Experimental techniques for imaging and measuring transient vapor nanobubbles," Appl. Phys. Lett. 101(26), 264102 (2012).

37. E. Y. Lukianova-Hleb et al., "Influence of transient environmental photothermal effects on optical scattering by gold nanoparticles," Nano Lett. 9(5), 2160-2166 (2009).

38. S. J. Sheldon et al., "Laser-induced thermal lens effect-a new theoretical-model," Appl. Opt. 21(9), 1663-1669 (1982).

39. A. Vogel et al., "Cavitation bubble dynamics and acoustic transient generation in ocular surgery with pulsed neodymium: YAG lasers," Ophthalmology 93(10), 1259-1269 (1986).

40. A. Vogel et al., "Energy balance of optical breakdown in water at nanosecond to femtosecond time scales," Appl. Phys. B 68(2), 271-280 (1999).

41. E. Boulais et al., "Plasma mediated off-resonance plasmonic enhanced ultrafast laser-induced nanocavitation," Nano Lett. 12(9), 4763-4769 (2012).

42. E. Y. Lukianova-Hleb et al., "Laser pulse duration is critical for the generation of plasmonic nanobubbles," Langmuir 30(25), 7425-7434 (2014).

43. W. Haiss et al., "Determination of size and concentration of gold nanoparticles from UV-Vis spectra," Anal. Chem. 79(11), 4215-4221 (2007).

44. P. K. Jain et al., "Calculated absorption and scattering properties of gold nanoparticles of different size, shape, and composition: applications in biological imaging and biomedicine," J. Phys. Chem. B 110(14), 7238-7248 (2006).
45. P. B. Johnson et al., "Optical constants of noble metals," Phys. Rev. B 6(12), 4370-4379 (1972).

46. J. W. Liaw et al., "Wavelength-dependent faraday-tyndall effect on laser-induced microbubble in gold colloid," J. Quant. Spectrosc. Radiat. Transfer 113(17), 2234-2242 (2012).

47. S. Hashimoto, "Studies on the interaction of pulsed lasers with plasmonic gold nanoparticles toward light manipulation, heat management, and nanofabrication," J. Photochem. Photobiol. C 13(1), 28-54 (2012).

48. S. V. Egerev et al., "Laser dynamic optoacoustic diagnostics of condensed media," Sov. Phys. Usp. 33(9), 739-762 (1990).

49. W. C. Vogt et al. "Size-dependent thresholds for melting and nanobubble generation using pulsed-laser irradiated gold nanoparticles," Proc. SPIE 10509, 105090C (2018).

50. Z. Y. Fang et al., "Evolution of light-induced vapor generation at a liquid-immersed metallic nanoparticle," Nano Lett. 13(4), 1736-1742 (2013).

51. E. Y. Lukianova-Hleb et al., "Plasmonic nanobubbles as transient vapor nanobubbles generated around plasmonic nanoparticles," ACS Nano 4(4), 2109-2123 (2010).

52. J. Lombard et al., "Kinetics of nanobubble generation around overheated nanoparticles," Phys. Rev. Lett. 112(10), 105701 (2014).

53. A. Siems et al., "Thermodynamics of nanosecond nanobubble formation at laser-excited metal nanoparticles," New J. Phys. 13(4), 043018 (2011).

Siqi Wang is currently working as a PhD candidate at Xi'an Jiaotong University, China. She has devoted her efforts to the study of the inherent physical mechanism of pulsed laser-excited micronanoscale bubbles, including the photothermal, photomechnical, and photoacoustic effects for optimization of laser-mediated therapy and diagnosis.

Zhenxi Zhang received his $\mathrm{PhD}$ in biomedical engineering and instrumentation from Xi'an Jiaotong University in 1990. Currently, he is a full professor and superintendent at the Institute of Biomedical Analytical Technology and Instrumentation, Xi'an Jiaotong University. He has devoted his efforts to the research of laser-based diagnosis and treatment of human disease, including nanoparticle-facilitated photothermal/photodynamic theranostics in cellular surgery based on the hyperspectral imaging and photoacoustic detection.

Biographies of the other authors are not available. 